\title{
Visible-to-near-infrared octave spanning supercontinuum generation in a silicon nitride waveguide
}

\author{
Haolan Zhao, ${ }^{1,2, *}$ Bart Kuyken, ${ }^{1,2}$ Stéphane Clemmen, ${ }^{1,2}$ François \\ Leo, ${ }^{1,2}$ Ananth Subramanian, ${ }^{1,2}$ Ashim Dhakal, ${ }^{1,2}$ Philippe Helin, ${ }^{3}$ Simone \\ Severi, ${ }^{3}$ Edouard Brainis, ${ }^{2,4}$ Gunther Roelkens, ${ }^{1,2}$ and Roel Baets ${ }^{1,2}$ \\ ${ }^{1}$ Photonics Research Group, Department of Information Technology, \\ Ghent University-imec, Sint-Pietersnieuwstraat 41, 9000 Ghent, Belgium \\ ${ }^{2}$ Center for Nano- and Biophotonics (NB-Photonics), \\ Ghent University, Sint-Pietersnieuwstraat 41, 9000 Ghent, Belgium \\ ${ }^{3}$ imec, Kapeldreef 75, B-3001 Leuven, Belgium \\ ${ }^{4}$ Physics and Chemistry of Nanostructures Group, \\ Ghent University, Krijgslaan 281-S3, 9000 Ghent, Belgium
}

compiled: April 10, 2015

\begin{abstract}
The generation of an octave spanning supercontinuum covering $488 \mathrm{~nm}$ to $978 \mathrm{~nm}$ (at $-30 \mathrm{~dB}$ ) is demonstrated for the first time on-chip. This result is achieved by dispersion engineering a $1 \mathrm{~cm}$ long $\mathrm{Si}_{3} \mathrm{~N}_{4}$ waveguide and pumping it with an $100 \mathrm{fs}$ Ti:Sapphire laser emitting at $795 \mathrm{~nm}$. This work offers a bright broadband source for biophotonic applications and frequency metrology.

OCIS codes: (320.6629) Supercontinuum Generation; (190.4390) Nonlinear optics, integrated optics. http://dx.doi.org/10.1364/XX.99.099999
\end{abstract}

Over the last decade, the progress of supercontinuum (SC) generation in photonic crystal fibers [1-3] has led to a series of advancements in spectroscopy [4], optical coherence tomography [5] and precise frequency metrology [6]. Recently SC generation on integrated CMOS compatible waveguide platforms has been attracting significant attention. Previous efforts mostly aimed at the telecom wavelength window [7] for WDM communication and at the mid-infrared range [8] for spectroscopic sensing. However, a SC covering the red to near-infrared spectral window where tissue and cells possess low absorption and scattering coefficients could offer particular advantages for biological applications such as bioimaging [9] and Raman spectroscopy [10, 11].

To produce a SC below $1 \mu \mathrm{m}$ on a CMOS-compatible integrated platform, there are two main hurdles to overcome: transparency of the waveguide and phase matching. The first one can be addressed by using silicon nitride as the waveguide material rather than silicon [12]. Recently a SC down to $665 \mathrm{~nm}$ has been obtained using a silicon nitride waveguide pumped at $1335 \mathrm{~nm}$ [13]. The second hurdle is to obtain anomalous dispersion required for efficient $\mathrm{SC}$ generation in $\mathrm{Si}_{3} \mathrm{~N}_{4}$ waveguides.

\footnotetext{
* Corresponding author: haolan.zhao@ugent.be
}

At visible wavelengths, this is not trivial because $\mathrm{Si}_{3} \mathrm{~N}_{4}$ possesses a strong normal material dispersion due the proximity to the material bandgap. The strong normal material dispersion thus needs to be compensated by the waveguide dispersion. A frequency comb using a silicon nitride microcavity has been demonstrated with comb lines down to $765 \mathrm{~nm}$ circumventing this requirement by using a combination of $\chi(2)$ and $\chi(3)$ nonlinear processes [14] yet it requires careful dispersion management. In this letter, inspired by the work on silicon platform $[15,16]$, we implement a similar method to achieve anomalous dispersion in a silicon nitride waveguide by partially underetching the silicon oxide underneath the $\mathrm{Si}_{3} \mathrm{~N}_{4}$ waveguide core. We demonstrate an $\mathrm{SC}$ ranging from $488 \mathrm{~nm}$ to $978 \mathrm{~nm}$ when pumping the waveguide at $795 \mathrm{~nm}$. To the best of our knowledge, this is the first demonstration of an octave spanning supercontinuum extending in the sub-500 $\mathrm{nm}$ wavelength range on an integrated platform. It constitutes a first step to a fully integrated broadband source for e.g. biophotonic applications.

The waveguide used in the experiment is fabricated in a CMOS pilot line at imec [17]. A $300 \mathrm{~nm}$-thick silicon nitride film is grown via low-pressure chemical vapor deposition (LPCVD) on a $1.6 \mu \mathrm{m}$ buried silicon oxide layer (BOX). A $500 \mathrm{~nm}$ wide waveguide of $1 \mathrm{~cm}$ long is then defined in the thin film using deep UV lithography and 

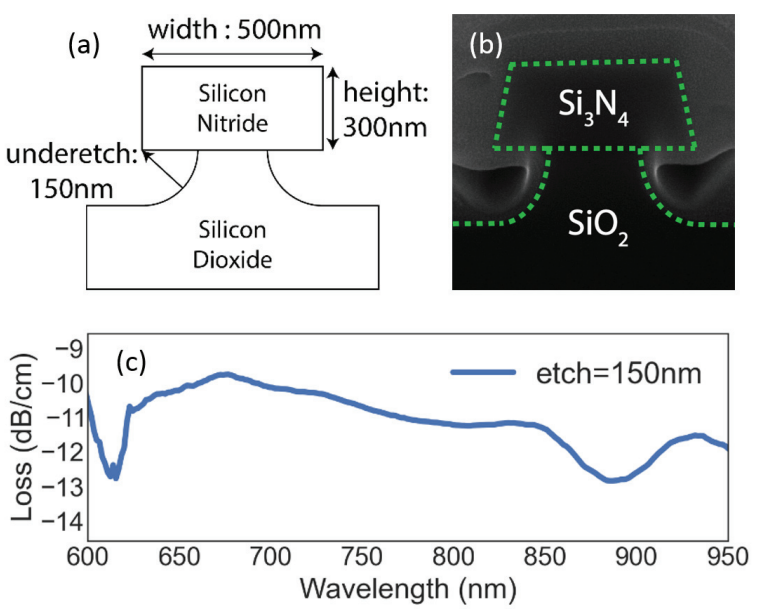

Fig. 1. (a) Schematic cross-section of the waveguide. (b) SEM picture of the waveguide cross-section. The material around the silicon nitride core is deposited for FIB crosssection. (c) The waveguide propagation loss from $600 \mathrm{~nm}$ to $950 \mathrm{~nm}$.

dry etching. The air-clad waveguide is then dipped into diluted $\mathrm{HF}$, which partially etches the oxide underneath it. Given the high etching selectivity between $\mathrm{SiO}_{2}$ and LPCVD $\mathrm{Si}_{3} \mathrm{~N}_{4}$ (200:1), the $\mathrm{Si}_{3} \mathrm{~N}_{4}$ waveguide dimensions are quasi-conserved. A schematic cross-section of the waveguide is shown in Fig. 1(a) while an SEM picture is shown in Fig. 1(b). The waveguide propagation loss after underetching is measured with a commercial supercontinuum source (NKT superK EXR-4) in a cutback measurement and the result is shown in Fig. 1(c). The waveguide loss from $650 \mathrm{~nm}$ to $850 \mathrm{~nm}$ is about $11 \mathrm{~dB} / \mathrm{cm}$. The increased index-contrast that is obtained by removing the oxide allows for increased confinement and hence stronger waveguide dispersion, compensating for the strong material dispersion of silicon nitride at visible wavelengths. Taking into account the material dispersion, the dependence of group velocity dispersion (GVD) on wavelength for the fundamental quasi-TE mode of the engineered waveguide is obtained via a full vectorial mode solver and the result is shown in Fig. 2. We can readily observe that an increase in the underetching results in an increased anomalous GVD. The waveguide we design for SC generation has a etching depth of $150 \mathrm{~nm}$, carrying an anomalous dispersion between $740 \mathrm{~nm}$ and $907 \mathrm{~nm}$ and the GVD of final waveguide lies in the shaded area due to fabrication uncertainty. The increased confinement also enhances the nonlinearity of the waveguide by reducing the mode area such that we obtain an effective nonlinearity of $7 \mathrm{~W}^{-1} \mathrm{~m}^{-1}$ at $795 \mathrm{~nm}$ by assuming a nonlinear index $n_{2}=24 \times 10^{-20} \mathrm{~m}^{2} / \mathrm{W}[18]$.

Fig. 3 shows the schematic experimental setup for $\mathrm{SC}$ generation. We use a pulse train generated by a Ti:Sapphire laser (Mai Tai, Spectra-Physics) operating at $795 \mathrm{~nm}$ as a pump. The pulses have a nominal squared

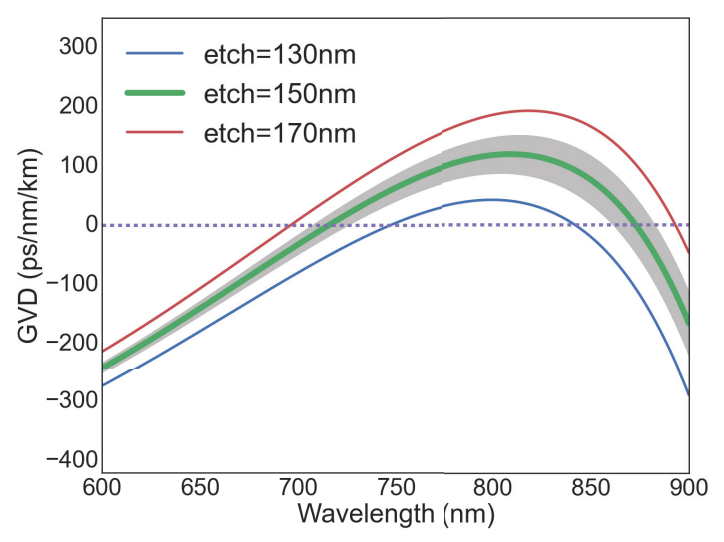

Fig. 2. Group velocity dispersion (GVD) of the underetched $\mathrm{Si}_{3} \mathrm{~N}_{4}$ waveguide for various values of the waveguide underetching. The GVD of waveguide used in the experiment lies in the shaded area.

hyperbolic secant shape in the temporal domain with a FWHM of 100 fs and a repetition rate of $80 \mathrm{MHz}$. To ensure the excitation of the fundamental TE mode in waveguide, a combination of a half-wave plate and a polarizing beam splitter is used. The pump is coupled into the waveguide using a microscope objective (40X, $\mathrm{NA}=0.5)$ and the generated spectrum is coupled out using a lensed fiber $(\mathrm{NA}=0.2)$. The coupling loss at the input is measured to be $8.5 \mathrm{~dB}$.

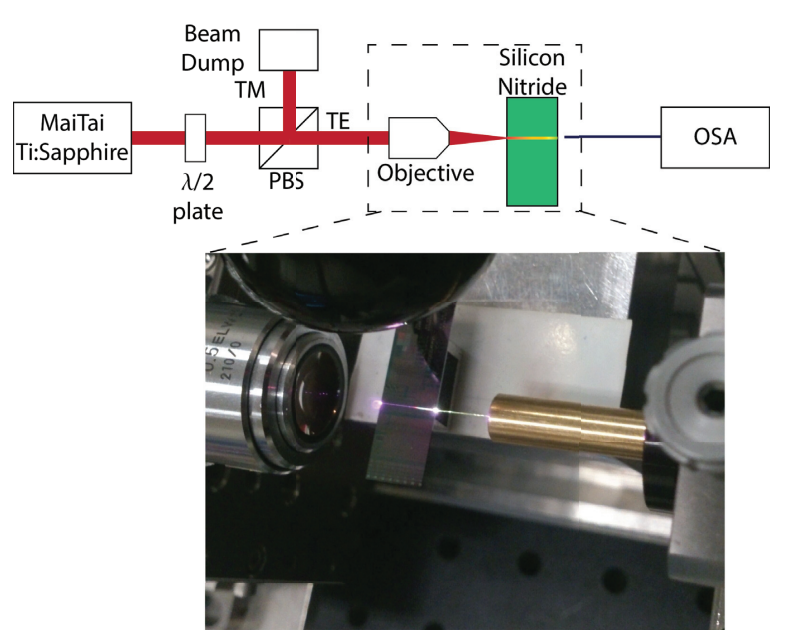

Fig. 3. Setup of the experiment. Visible light generation from the end of the chip can readily be observed.

We use an optical spectrum analyzer (450 nm - 1050 $\mathrm{nm}$, Advantest Q8381A) to record the generated spectrum. Fig. 4 shows the measured spectra at various input peak powers coupled into the waveguide. At a low peak power of $62 \mathrm{~W}$ the spectral width is the same as the input pulse. At $182 \mathrm{~W}$, small ripples emerge in the center of the pulse, which is attributed to self-phase modulation (SPM). At $491 \mathrm{~W}$ we see clearly two peaks 
emerging at $917 \mathrm{~nm}$ and $495 \mathrm{~nm}$. Since the peaks appear at a wavelength where the waveguide has normal dispersion, we attribute these two peaks to dispersive waves. When the power is further increased the dispersive wave centered at $917 \mathrm{~nm}$ red-shifts to $937 \mathrm{~nm}$ while the one at $495 \mathrm{~nm}$ retains its original spectral position. At the maximal available coupled power of $874 \mathrm{~W}$, the $-30 \mathrm{~dB}$ bandwidth spans $490 \mathrm{~nm}$ from $488 \mathrm{~nm}$ to 978 $\mathrm{nm}$, slightly more than an octave. From Fig. 3 one can clearly see visible red light generated right after the input and its intensity increasing along the propagation direction. Further increasing the power would help improve the spectral homogeneity yet the limitation is the damage occurring at the input facet of the waveguide. To ensure no spectral broadening takes place in the collection fiber, we deliberately misaligned the lensed fiber both vertically and horizontally at the output facet to reduce the spectrum recorded in OSA by $20 \mathrm{~dB}$ at the same coupling angle and we observe no change in the shape of the spectrum.

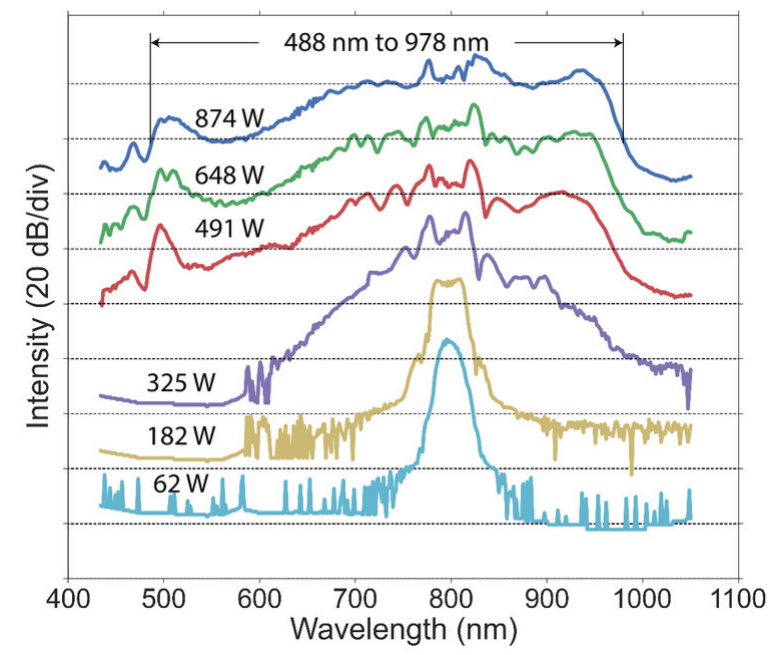

Fig. 4. Output spectra of the waveguide at various input peak power coupled into the waveguide. The spectra are shifted vertically for clarity.

To gain insight in the physical mechanism behind the spectral broadening, we model the SC generation in our waveguide with the Nonlinear Schrodinger Equation (NLSE). This equation describes the evolution of the temporal envelop of the electric field $E(z, t)$ inside the nonlinear medium, taking into account the propagation loss, SPM, GVD and self-steepening effects. The equation reads [1]

$$
\begin{aligned}
\frac{\partial E(z, t)}{\partial z} & =i \sum_{k \geq 2} i^{k} \frac{\beta_{k}}{k !} \frac{\partial^{k} E}{\partial t^{k}}-\frac{\alpha_{l}}{2} E \\
& +i \gamma\left(|E|^{2} E+\frac{i}{\omega_{p}} \frac{\partial}{\partial t}\left(|E|^{2} E\right)\right)
\end{aligned}
$$

Here $\beta_{k}$ is the $k$-th order chromatic dispersion ob- tained by Taylor series expansion of the wave number $\beta, \alpha_{l}$ is the linear loss of the waveguide, $\omega_{0}$ is the pump frequency and $\gamma$ is the nonlinear coefficient of the waveguide. The Raman term is omitted in the equation since its contribution it rather limited. $[19,20]$
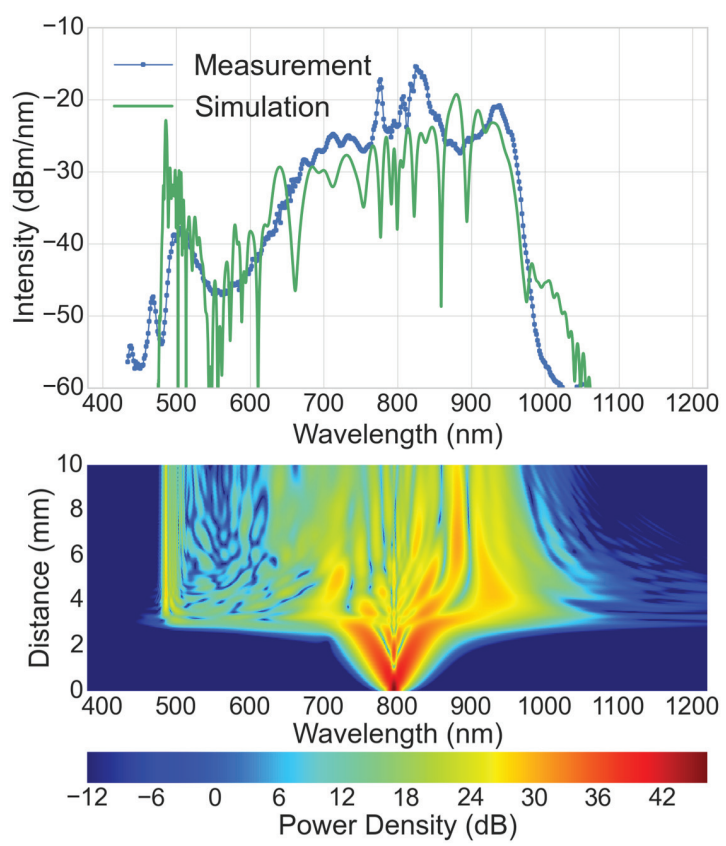

Fig. 5. (Top) Comparison between measured spectrum and simulation in the output and (below) evolution of the pulse inside waveguide with $874 \mathrm{~W}$ input peak power.

The NLSE is numerically solved with the split-step Fourier algorithm using the chromatic dispersion up to the 6 th order and we have plug the spectral dependence of mode area into calculation. Fig. 5 shows the evolution of the pulse inside the waveguide. Because the waveguide dimensions determined by SEM picture involves a certain degree of uncertainty $(\sim 15 \mathrm{~nm})$, the dispersions are determined by fitting the experimental data with boundary conditions as shown as the shaded area in Fig. 2. The top panel plots the output pulse spectrum of both the simulation and the measurement, which reveals a reasonable agreement in terms of the general shape. The spectral width and the positions of the two dispersive waves in the experiment are reproduced by the simulation. The simulation accounts for a slight chirp of the input pulse of 0.15 extracted by fitting output spectrum at $182 \mathrm{~W}$. It is noticeable that the predicted peak power at $495 \mathrm{~nm}$ is around $8 \mathrm{~dB}$ higher than the measurement, which we attribute to a combination of higher scattering loss and stronger material absorption at shorter wavelength. The increased loss is reflected in a measurement with $445 \mathrm{~nm}$ laser (Wicked Laser, Spyder 3) where we found the waveguide becomes so lossy that no light could transmit through.

The position of the dispersive waves can also be pre- 
dicted by the phase-matching condition [1]

$$
\beta\left(\omega_{\mathrm{DW}}\right)-\frac{\omega_{\mathrm{DW}}}{v_{\mathrm{g}, \mathrm{s}}}=\beta\left(\omega_{\mathrm{s}}\right)-\frac{\omega_{\mathrm{s}}}{v_{\mathrm{g}, \mathrm{s}}}+\frac{\gamma P_{\mathrm{s}}}{2} .
$$

Here $\beta$ is the wave number, $\gamma$ is the nonlinear coefficient, $v_{\mathrm{g}, \mathrm{s}}$ is the soliton group velocity, $P_{\mathrm{s}}$ is the soliton peak power, and $\omega_{\mathrm{DW}}$ and $\omega_{\mathrm{s}}$ are the frequency of dispersive wave and soliton respectively. By using a peak soliton power of $874 \mathrm{~W}$, the dispersive waves are predicted to appear at $934 \mathrm{~nm}$ and $498 \mathrm{~nm}$, which is in agreement with the experiment. The deviations between simulation and measurement are attributed to uncertainty of waveguide dimensions. With the phasematching condition, the red-shift of the near-infrared dispersive wave can be readily explained by the variation in soliton power. We believe the calculation based on the phase-matching condition and the numerical simulation results confirm the origin of the peaks.

In conclusion, we have demonstrated an octave spanning SC covering a large part of the visible spectrum in a partially underetched dispersion engineered silicon nitride waveguide. To the best of our knowledge this work constitutes the first visible $\mathrm{SC}$ on an integrated platform, offering a novel compact visible broadband source for Raman spectroscopy, confocal microscopy, optical coherence tomography and precise frequency metrology. Our result also shows one can tailor the zero dispersion wavelength to the visible wavelength region in the $\mathrm{Si}_{3} \mathrm{~N}_{4}$ platform, offering further opportunities for nonlinear optics at visible wavelengths.

The work is supported by the FP7-ERC-InSpectra advanced grant.

\section{References}

[1] J. M. Dudley, G. Genty, and S. Coen, "Supercontinuum generation in photonic crystal fiber," Rev. Mod. Phys. 78, 1135-1184 (2006).

[2] C. R. Petersen, U. Mller, I. Kubat, B. Zhou, S. Dupont, J. Ramsay, T. Benson, S. Sujecki, N. Abdel-Moneim, Z. Tang, D. Furniss, A. Seddon, and O. Bang, "Midinfrared supercontinuum covering the $1.413 .3 \mathrm{~m}$ molecular fingerprint region using ultra-high na chalcogenide step-index fibre," Nat Photon 8, 830-834 (2014).

[3] M.-T. Tsai and M.-C. Chan, "Simultaneous 0.8, 1.0, and $1.3 \mu \mathrm{m}$ multispectral and common-path broadband source for optical coherence tomography," Opt. Lett. 39, 865-868 (2014).

[4] K. Lindfors, T. Kalkbrenner, P. Stoller, and V. Sandoghdar, "Detection and spectroscopy of gold nanoparticles using supercontinuum white light confocal microscopy," Phys. Rev. Lett. 93, 037401 (2004).

[5] R. Leitgeb, W. Drexler, A. Unterhuber, B. Hermann, T. Bajraszewski, T. Le, A. Stingl, and A. Fercher, "Ultrahigh resolution fourier domain optical coherence tomography," Opt. Express 12, 2156-2165 (2004).

[6] T. Udem, R. Holzwarth, and T. W. Hänsch, "Optical frequency metrology," Nature 416, 233-237 (2002).

[7] J. Safioui, F. Leo, B. Kuyken, S.-P. Gorza, S. K. Selvaraja, R. Baets, P. Emplit, G. Roelkens, and S. Mas- sar, "Supercontinuum generation in hydrogenated amorphous silicon waveguides at telecommunication wavelengths," Opt. Express 22, 3089-3097 (2014).

[8] B. Kuyken, X. Liu, R. M. Osgood Jr., R. Baets, G. Roelkens, and W. Green, "Mid-infrared to telecomband supercontinuum generation in highly nonlinear silicon-on-insulator wire waveguides," Opt. Express 19, 20172-20181 (2011).

[9] H. Yokoyama, H. Tsubokawa, H. Guo, J.-i. Shikata, K.-i. Sato, K. Takashima, K. Kashiwagi, N. Saito, H. Taniguchi, and H. Ito, "Two-photon bioimaging utilizing supercontinuum light generated by a high-peakpower picosecond semiconductor laser source," Journal of Biomedical Optics 12, 054019-054019-5 (2007).

[10] Q. Xu, K. Shi, and Z. Liu, "Time-resolved coherent antistokes raman spectroscopy impulsively excited by supercontinuum," Journal of Raman Spectroscopy 42, 21002104 (2011).

[11] H. Mikami, M. Shiozawa, M. Shirai, and K. Watanabe, "Compact light source for ultrabroadband coherent antistoke raman scattering (cars) microscopy," Opt. Express 23, 2872-2878 (2015).

[12] L. Zhang, Y. Yan, Y. Yue, Q. Lin, O. Painter, R. G. Beausoleil, and A. E. Willner, "On-chip two-octave supercontinuum generation by enhancing self-steepening of optical pulses," Opt. Express 19, 11584-11590 (2011).

[13] R. Halir, Y. Okawachi, J. S. Levy, M. A. Foster, M. Lipson, and A. L. Gaeta, "Ultrabroadband supercontinuum generation in a cmos-compatible platform," Opt. Lett. 37, 1685-1687 (2012).

[14] S. Miller, K. Luke, Y. Okawachi, J. Cardenas, A. L. Gaeta, and M. Lipson, "On-chip frequency comb generation at visible wavelengths via simultaneous secondand third-order optical nonlinearities," Opt. Express 22, 26517-26525 (2014).

[15] F. Leo, U. Dave, S. Keyvaninia, B. Kuyken, and G. Roelkens, "Measurement and tuning of the chromatic dispersion of a silicon photonic wire around the half band gap spectral region," Opt. Lett. 39, 711-714 (2014).

[16] L. Yin, Q. Lin, and G. P. Agrawal, "Soliton fission and supercontinuum generation in silicon waveguides," Opt. Lett. 32, 391-393 (2007).

[17] A. Subramanian, P. Neutens, A. Dhakal, R. Jansen, T. Claes, X. Rottenberg, F. Peyskens, S. Selvaraja, P. Helin, B. Dubois, K. Leyssens, S. Severi, P. Deshpande, R. Baets, and P. Van Dorpe, "Low-loss singlemode pecvd silicon nitride photonic wire waveguides for 532 - $900 \mathrm{~nm}$ wavelength window fabricated within a cmos pilot line," Photonics Journal, IEEE 5, 22028092202809 (2013).

[18] K. Ikeda, R. E. Saperstein, N. Alic, and Y. Fainman, "Thermal and kerr nonlinear properties of plasmadeposited silicon nitride/ silicon dioxide waveguides," Opt. Express 16, 12987-12994 (2008).

[19] A. Dhakal, A. Z. Subramanian, P. Wuytens, F. Peyskens, N. L. Thomas, and R. Baets, "Evanescent excitation and collection of spontaneous raman spectra using silicon nitride nanophotonic waveguides," Opt. Lett. 39, 4025-4028 (2014).

[20] A. Dhakal, P. Wuytens, F. Peyskens, A. Z. Subramanian, N. Le Thomas, and R. Baets, "Silicon-nitride waveguides for on-chip raman spectroscopy," (2014), vol. 9141, pp. 91411C-91411C-7. 